\title{
Response Assessment with MRI after Chemoradiotherapy in Rectal Cancer: Current Evidences
}

\author{
Nieun Seo, MD ${ }^{1 *}$, Honsoul Kim, MD ${ }^{2 *}$, Min Soo Cho, MD ${ }^{3}$, Joon Seok Lim, MD \\ Departments of ${ }^{1}$ Radiology and ${ }^{3}$ Surgery, Severance Hospital, Yonsei University College of Medicine, Seoul, Korea; ${ }^{2}$ Department of Radiology, \\ Samsung Medical Center, Sungkyunkwan University School of Medicine, Seoul, Korea
}

Baseline magnetic resonance imaging (MRI) has become the primary staging modality for surgical plans and stratification of patient populations for more efficient neoadjuvant treatment. Patients who exhibit a complete response to chemoradiotherapy (CRT) may achieve excellent local tumor control and better quality of life with organ-preserving treatments such as local excision or even watch-and-wait management. Therefore, the evaluation of tumor response is a key factor for determining the appropriate treatment following CRT. Although post-CRT MRI is generally accepted as the first-choice method for evaluating treatment response after CRT, its application in the clinical decision process is not fully validated. In this review, we will discuss various oncologic treatment options from radical surgical technique to organ-preservation strategies for achieving better cancer control and improved quality of life following CRT. In addition, the current status of post-CRT MRI in restaging rectal cancer as well as the main imaging features that should be evaluated for treatment planning will also be described for the tailored treatment.

Keywords: Rectal cancer; Magnetic resonance imaging; Pathological complete response; Neoadjuvant chemoradiotherapy; Tumor response

\section{INTRODUCTION}

Over the past 30 years, the introduction of preoperative chemoradiotherapy (CRT), advances in surgical techniques, and new imaging modalities for selecting treatment and surgical planning have resulted in dramatic improvements in local recurrence rates and quality of life in patients with rectal cancer. In this context, baseline magnetic resonance imaging (MRI) in patients with locally advanced rectal cancer (LARC) has an undeniable role in the determination of neoadjuvant CRT through risk stratification by the evaluation of circumferential resection margin (CRM),

Received September 5, 2018; accepted after revision April 7, 2019. *These authors contributed equally to this work.

Corresponding author: Joon Seok Lim, MD, Department of Radiology, Severance Hospital, Yonsei University College of Medicine, 50-1 Yonsei-ro, Seodaemun-gu, Seoul 03722, Korea.

- Tel: (822) 2228-7400 - Fax: (822) 2227-8337

- E-mail: jslim1@yuhs.ac

This is an Open Access article distributed under the terms of the Creative Commons Attribution Non-Commercial License (https://creativecommons.org/licenses/by-nc/4.0) which permits unrestricted non-commercial use, distribution, and reproduction in any medium, provided the original work is properly cited.
T3 sub-classifications, and assessment of extramural vessel invasion, nodal status, and sphincter invasion (1). Therefore, there is a uniform consensus on the value of baseline MRI in the preoperative evaluation for patients with rectal cancer (2). After CRT, most LARCs demonstrate variable degrees of tumor response, including pathologic complete remission ( $p C R$ ) in $4-31 \%$ of patients (3). Patients with complete remission (CR) by CRT can achieve excellent local tumor control and a better quality of life with organpreserving treatments such as local excision or even watchand-wait management (4). Therefore, an evaluation of tumor responses may become a key factor in determining the treatment strategies for individual patient after CRT.

Post-CRT MRI is also considered to be the first choice for evaluating treatment response after CRT. However, whether post-CRT MRI evaluation for tumor response can inform treatment strategies remains controversial. Surgeons are often reluctant to deviate from initial plans because of concerns about residual viable tumor cells in the rectal wall or the mesorectal nodes, even in patients with clinical complete response (CCR) after preoperative CRT (5). Furthermore, the diagnostic outcomes and clinical 
impacts of post-CRT MRI have not been satisfactory for deciding the more radical surgical dissection or the organpreserving strategy because of insufficient reliability for differentiating residual tumors from CRT-related changes such as edema, necrosis, and particularly fibrotic change after CRT. Therefore, radiologists should be aware that their interpretation might result in a faulty decision for organpreserving treatment or conversely unnecessary radical surgery which compromises patients' quality of life.

In this review, we will introduce recent advances in the treatment of LARC and describe the role of post-CRT MRI in the clinical restaging of rectal cancer after CRT concerning improving local control and patient quality of life. Finally, we will discuss the issues faced by radiologists in determining tumor response after CRT.

\section{Treatment Options after Chemoradiotherpy for Locally Advanced Rectal Cancers}

Total mesorectal excision (TME) is the standard treatment for the majority of patients who underwent CRT for rectal cancer. It emphasizes en-bloc resection of the mesorectum which refers to the fatty tissue envelope of the rectum, containing blood and lymphatic vessels (6). TME can be performed as a component of low anterior resection (LAR) or abdominoperineal excision (APE) procedures. The decision to perform either LAR or APE is based on the tumor location in relation to the anal sphincter complex. LAR is any sphincter-preserving resection with anastomosis beneath the anterior peritoneal reflection. LAR can be further divided into LAR and ultra-LAR including intersphincteric resection based on the level of dissection and anastomosis (above or below the mesorectal end, respectively) (7). While LAR is performed for tumors that are distant from the anal sphincter, APE is defined as an enbloc removal of a tumor with the anal sphincter and anus, accompanying a permanent colostomy (Table 1, Fig. 1) and is usually performed for tumors that are near to or involve the anal sphincter (intersphincteric plane or external sphincter involvement). Therefore, the distance between the distal end of the tumor and anorectal junction should be provided in the post-CRT MRI evaluation. In the era of neoadjuvant CRT, distal surgical margin even $\leq 1 \mathrm{~cm}$ appear sufficient (8). Anatomically, there is no mesorectal fat up to approximately $1 \mathrm{~cm}$ above the anorectal junction (upper end of intersphincteric plane) (Fig. 2) (9). Treatment failure is common at this level because the tumor penetrating the muscle proper directly abuts levator ani muscle without intervening mesorectal fat $(10,11)$. Therefore, the information about whether the distal level of a tumor is located above or below the level of the mesorectal end (approximately upper end of intersphincteric plane) $1 \mathrm{~cm}$ above the anorectal junction is important for determining the salvage of anal sphincter.

APE can be classified into traditional APE or extralevator abdominoperineal excision (ELAPE) (Fig. 1). Unlike traditional APE, because ELAPE includes the en-bloc removal of the levator muscles, ELAPE is considered to be a more radical approach and thus increases CRM clearance, particularly in patients whose levator plane is still threatened on post-CRT MRI (Figs. 1, 3) (12). For T4b tumors which invade other organs such as urinary bladder, prostate, uterus, or sacrum, pelvic exenteration can be performed (13).

Recently, organ-preserving strategies have also been deployed to avoid rectal excision in patients in whom complete tumor shrinkage without residual tumor tissue has been identified after neoadjuvant CRT. These strategies are

Table 1. Relevant Post-CRT MRI Findings according to Type of Surgery

\begin{tabular}{ll}
\hline \multicolumn{1}{c}{ Type of Surgery } & \multicolumn{1}{c}{ Relevant Post-CRT MRI Findings } \\
\hline Low anterior or sphincter-saving resection & - Distal margin of treated tumor above $1 \mathrm{~cm}$ line above anorectal junction \\
APE or intersphincteric resection* & - Intact anal sphincter integrity \\
APE & - Distal margin of treated tumor below $1 \mathrm{~cm}$ line above anorectal junction \\
Extralevator abdominoperineal excision & - Intersphincteric space or external sphincter involvement \\
Pelvic exenteration & - Levator ani muscle plane threatening $(<1-2 \mathrm{~mm})$ or invasion \\
Local excision & - Adjacent organ invasion \\
Selective pelvic lymph node dissection & - Treated tumor confined to rectal wall without extramural tumor infiltration (ycT1) \\
\hline
\end{tabular}

* Intersphincteric resection can be performed in cases of no tumor involvement in intersphincteric space or external sphincter. APE $=$ abdominoperineal excision, $C R T$ = chemoradiotherapy, MRI = magnetic resonance imaging 


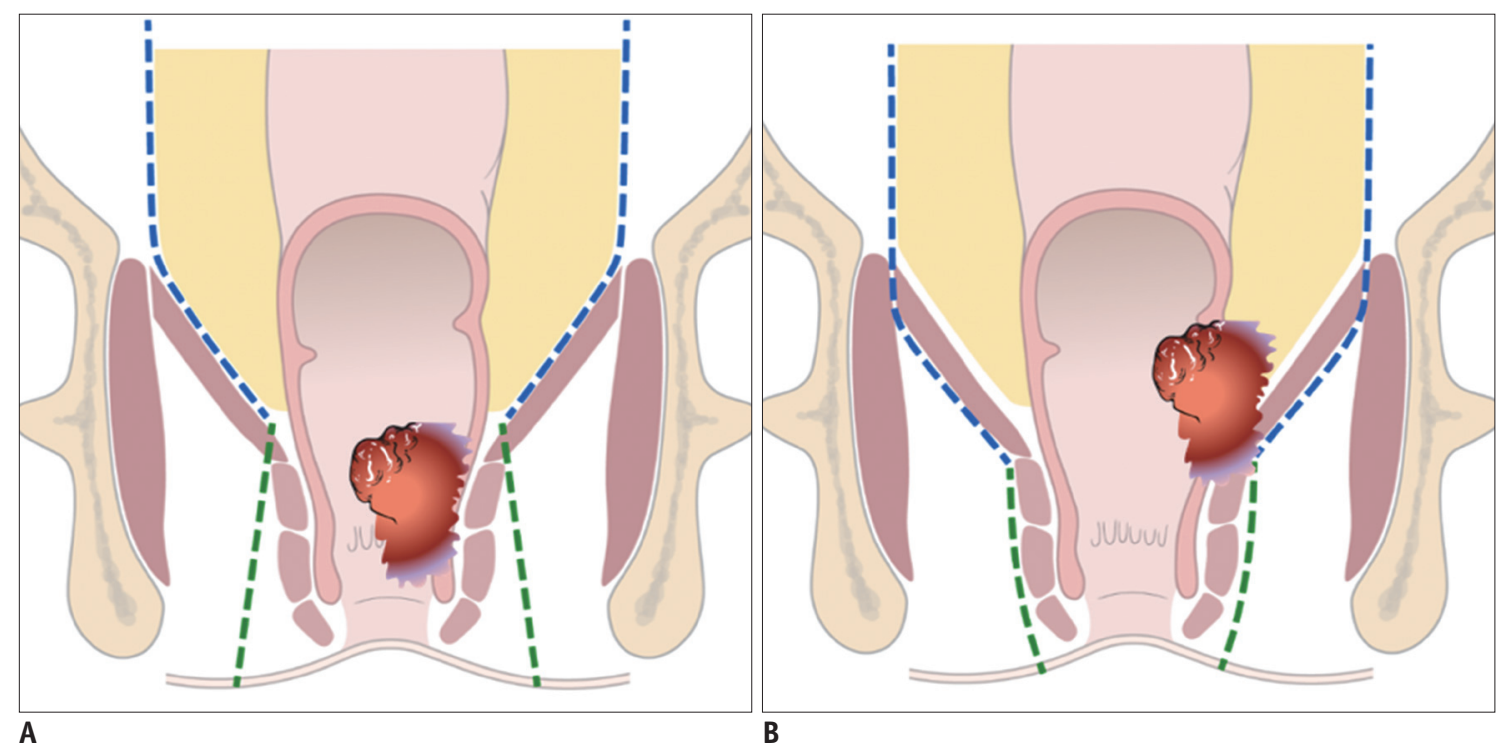

Fig. 1. Traditional APE and extralevator APE.

A. Traditional APE: Dotted line indicates dissection plane. Because levator muscle is not included in surgical specimen, it is appropriate for tumors without threatening to levator muscle plane. B. Extralevator APE: Tumor infiltration extends to left levator ani muscle. Levators are removed in this surgical specimen. This type of surgery is mandatory for lower rectal cancer with levator involvement to achieve negative pCRM. $\mathrm{APE}=$ abdominoperineal excision, $\mathrm{pCRM}=$ pathologic circumferential resection margin

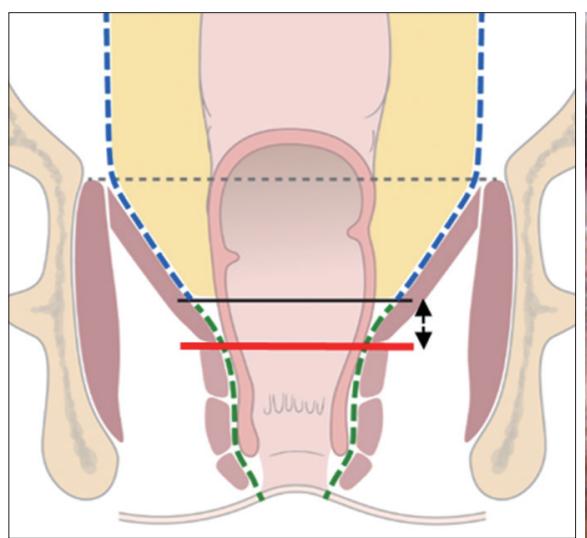

A

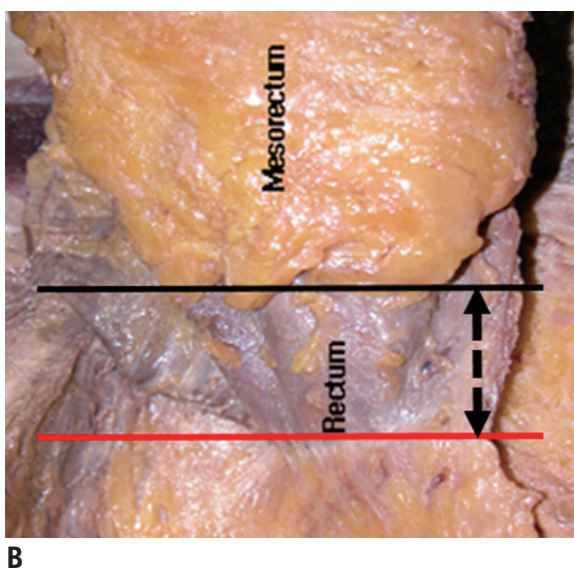

B

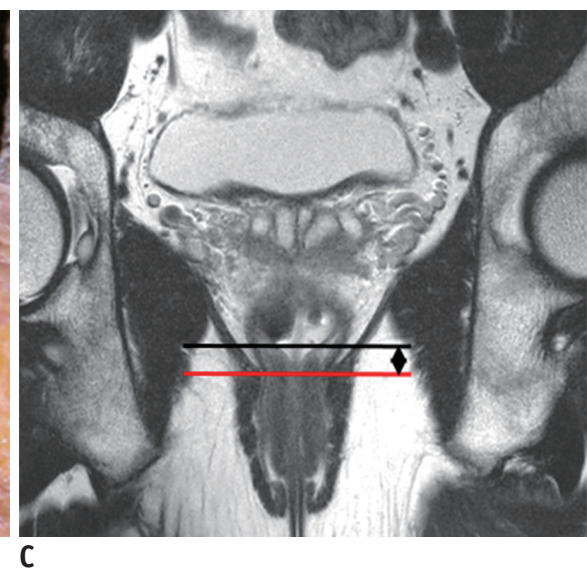

Fig. 2. Mesorectal fat bare area between distal end of mesorectum (black line) and level of anorectal junction (red line).

A. Schematic drawing depicting mesorectal fat bare area. Length of bi-directional arrow is approximately $1 \mathrm{~cm}$. B. Cadaveric dissection image shows that mesorectal fat is absent along longitudinal extent corresponding to same bi-directional arrow. At this site, only rectal wall is present. (Adapted from Kim. Yonsei Med J 2005;46:737-749) (73). C. On T2-weighted oblique coronal image, imaginary black line is drawn at $1 \mathrm{~cm}$ (bidirectional arrow) above anorectal junction level (red line) which roughly corresponds to upper end of intersphincteric plane.

divided into local excision and watch-and-wait strategy. Patients showing clinical downstaging to cT0-1N0 after CRT may be candidates for local excision. Local excision procedures include transanal excision (TAE), transanal endoscopic microsurgery (TEM), and transanal minimally invasive surgery (TAMIS) (14). TAE is indicated for tumors that lie below the level of peritoneal reflection. TEM or TAMIS are performed for tumors that lie above the peritoneal reflection. With these local excision techniques, resection of full tissue layers including mucosa, submucosa, and muscle proper can be performed. However, the complete dissection of the lymph nodes is impossible. Therefore, careful evaluation to guarantee the absence of extramural tumor infiltration and residual metastatic lymph node on post-CRT MRI is crucial for oncologic safety.

Surgical planning should be conducted to accurately assess the extent of the local tumor and the relationship between residual tumors and the anal sphincter on post-CRT MRI. However, distinguishing residual viable tumors from fibrotic changes following CRT is often challenging on post-CRT 


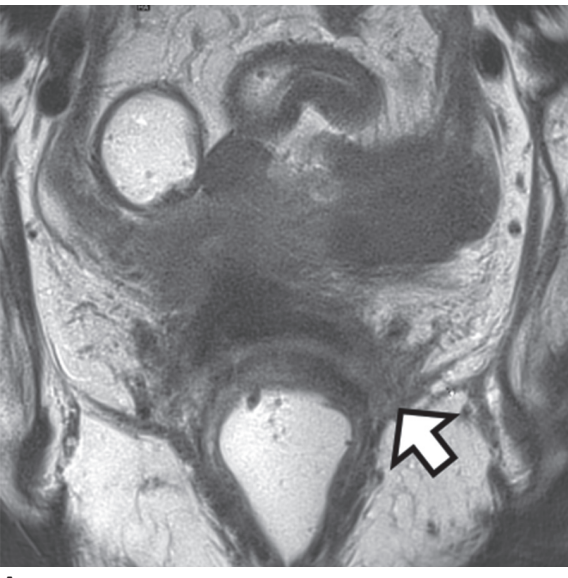

A

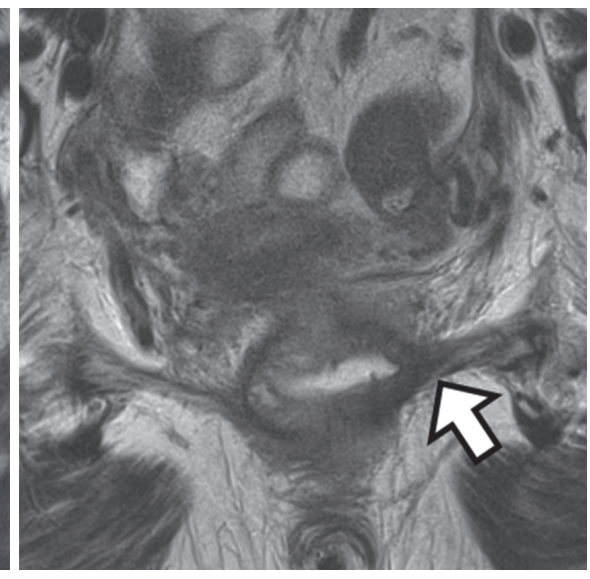

B

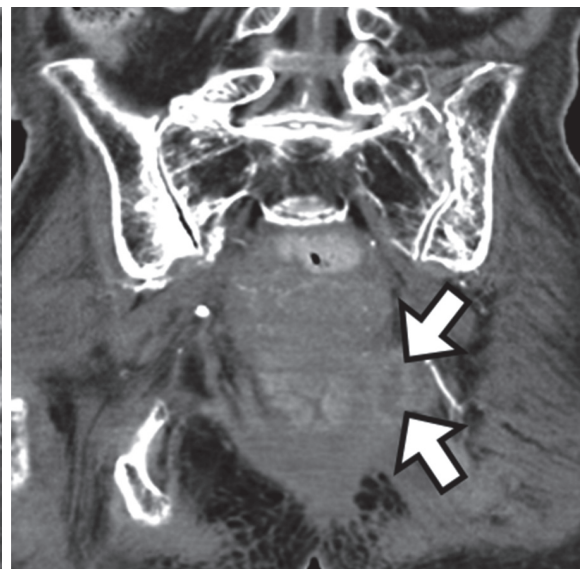

C

Fig. 3. Distal rectal cancer with left levator ani muscle involvement.

A. On baseline MRI, ulceroinfiltrative mass is observed in distal rectum with tumor infiltration into left levator ani muscle (arrow). B. On postCRT MRI, signal drop is seen within entire tumor. However, shrunken tumor still invades levator muscle (arrow). Traditional APE was performed. Histopathology revealed tumors with pathologic tumor regression grade 3 and positive pCRM (not shown). C. Follow-up CT 6 months after operation shows recurrent mass at remnant left levator muscle (arrows). CRT = chemoradiotherapy, MRI = magnetic resonance imaging

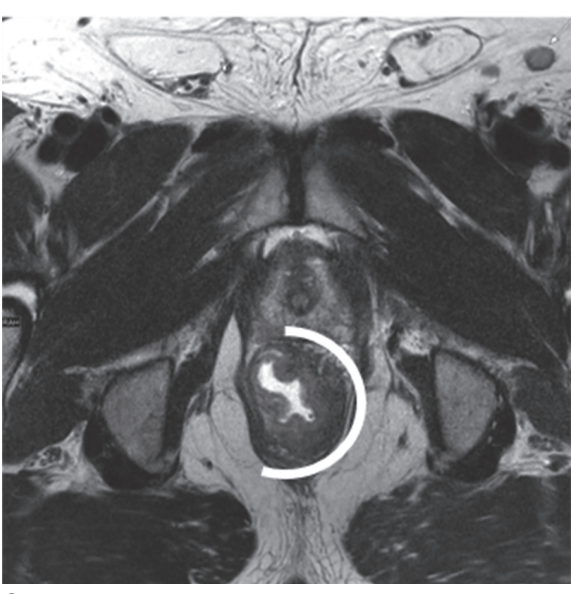

A

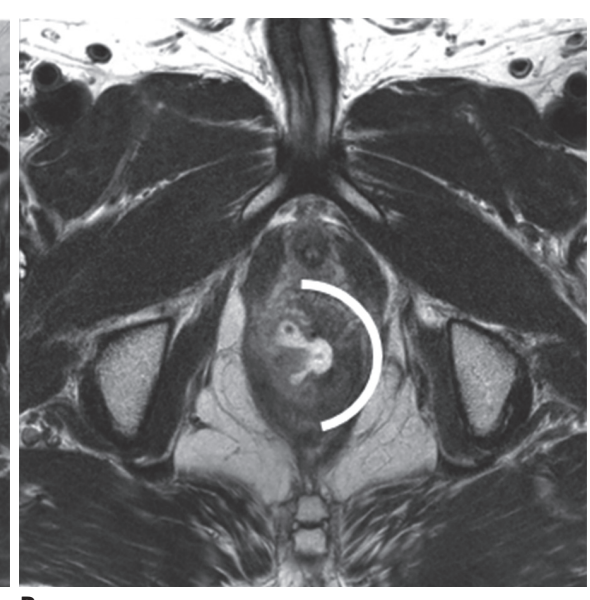

B

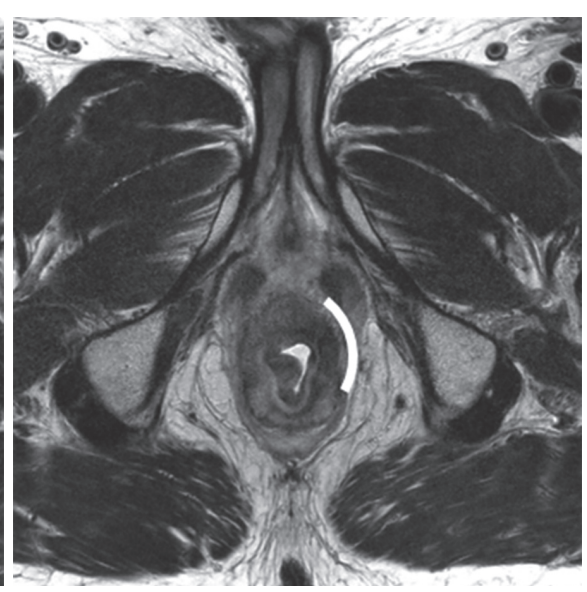

c

Fig. 4. Progressive tumoricidal effect and fibrotic change in rectal cancer after routine timing window.

A. On baseline MRI, semi-circular mass with intermediate SI is noted at left lateral wall of rectum (curved line indicates circumferential tumor extent). B. Post-CRT MRI obtained at four weeks after completion of CRT shows increased proportion of fibrotic change as well as decreased proportion of tumor SI, indicating tumor regression. Patient refused surgical option at that time. C. On post-CRT MRI obtained at 22 weeks, proportion of fibrotic change is further increased with decreased circumferential tumoral extent. Histopathology after subsequent APE revealed ypT2 (not shown). SI= signal intensity

MRI. Although fibrotic changes appear as decreased signal intensity (SI) on T2-weighted images, even the dense dark fibrotic tissue on MRI could have microscopic viable residual cancer cells. The possibility of a viable residual tumor in the dark fibrotic tissue is known to be approximately $50 \%$ (15). Determining tumor relationships with the surrounding structures (e.g., the anal sphincter) on the basis of T2 SI changes only according to the hypothesis that only the intermediate T2 SI portion within the treated mass is viable cancer and the dark SI portion is exclusively fibrotic change, may jeopardize oncologic safety (Fig. 3). Furthermore, the relationship between tumors and surrounding organs or sphincters on post-CRT MRI should be determined on the basis of the whole treated tumor including any fibrotic tissues, except for fibrotic spiculations. The possibility of residual cancer cells within the fibrotic spiculation on postCRT MRI is known to be extremely rare (16).

\section{Timing of MRI for Evaluation of Treatment Response after Chemoradiotherapy}

The optimal timing of post-CRT MRI for the evaluation of tumor response depends on the timing of surgery after CRT. However, surgical timing with respect to CRT varies 
due to a lack of consensus on the optimal interval. Because tumoricidal effects of CRT increase over time (Fig. 4), it is possible that more cases achieving PCR would be identified with a longer waiting time after CRT (17). However, because surgeons are reluctant to delay operation beyond eight weeks due to concerns about radiation-induced pelvic fibrosis and related surgical complications, surgery is generally performed at approximately $6-8$ weeks after the completion of CRT (18), although delaying surgery more than eight weeks after completion of CRT may be more beneficial (19). Therefore, post-CRT MRI should be performed 6-8 weeks after the termination of CRT, and immediately before surgery.

\section{Reported Items on Post-Chemoradiotherapy MRI}

After neoadjuvant treatment, reports of rectal MRI should include the following items: local tumor status, mesorectal fascia (MRF) involvement, lymph node involvement, and extramural venous invasion (EMVI) status (20).

\section{Local Tumor Status}

Accurate preoperative assessment of local tumor status following CRT is essential for deciding an appropriate treatment strategy. Tumor response after CRT can be evaluated by assessing MRI-based tumor regression grade (mrTRG) using an established scoring system and post-treatment T staging (ymrT) (21). Scoring systems established by the Magnetic Resonance Imaging and Rectal Cancer European Equivalence Study (MERCURY) study group and the European Society of Gastrointestinal and Abdominal Radiology (ESGAR) are mostly similar but differ most notably in their definitions of good responders (Table 2, Fig. 5) (20-22). mrTRG scores developed by the MERCURY study group were derived from pathologic TRG ( $p$ TRG). mrTRG was defined according to whether fibrous or tumor SI predominates within the entire tumor (23). In other words, mrTRG is determined by the proportion of presumed residual tumor and fibrotic change on T2-weighted images. On postCRT T2-weighted imaging, the fibrotic portion shows dark SI similar to that of the muscularis propria, whereas the portion of the residual tumor shows intermediate SI similar to that of baseline tumor (24). mrTRG scoring system and the definition of TRG 1-2 have been changed between 2012 and 2016 (20). Grade 1 now indicates a complete radiologic response with a linear/crescentic 1-2 $\mathrm{mm}$ scar in the mucosa or submucosa on MRI (Fig. 5B). Grade 2 is indicated by a good response and MRI findings include dense fibrosis without obvious residual tumor, suggesting minimal residual disease or no tumor (Fig. 5C). Grade 3 is indicated by a moderate response, with more than $50 \%$ of areas with fibrosis/mucin and visible intermediate tumor signal on MRI (Fig. 5D). Grade 4 suggests a slight response to treatment, with MRI findings of few areas with fibrosis/ mucin and mostly tumor-derived MRI signal (Fig. 5E).

Grade 5 indicates no response to therapy, showing a similar tumor to the baseline or significant tumor regrowth. These mrTRG scores have significant implications for clinical care and outcomes. According to a previous study, disease-free survival and overall survival differed significantly between mrTRG 1-3 (good response) and mrTRG 4-5 (poor response) (25). Recently, the definition of good responder has been evolved in mrTRG scoring system due to an introduction of organ-preserving approach. It requires a higher positive predictive value (PPV) for $p C R$ or near-pCR. Therefore, recent

Table 2. MRI-Based Tumor Regression Grade

\begin{tabular}{|c|c|c|c|c|}
\hline Grade & Response & MERCURY (2012) & MERCURY (2016) & ESGAR (2016) \\
\hline mrTRG 1 & $\begin{array}{l}\text { Complete } \\
\text { response }\end{array}$ & $\begin{array}{l}\text { No evidence of tumor SI or } \\
\text { fibrosis only }\end{array}$ & $\begin{array}{l}\text { Linear/crescentic } 1-2 \mathrm{~mm} \text { scar } \\
\text { in mucosa or submucosa only }\end{array}$ & $\begin{array}{l}\text { Completely normalized } \\
\text { rectal wall }\end{array}$ \\
\hline mrTRG 2 & $\begin{array}{l}\text { Good } \\
\text { response }\end{array}$ & $\begin{array}{l}\text { Dense hypointense fibrosis } \\
\text { with minimal residual tumor }\end{array}$ & $\begin{array}{l}\text { No obvious residual tumor, } \\
\text { signifying minimal residual disease } \\
\text { or no tumor }\end{array}$ & $\begin{array}{l}\text { Fibrotic wall thickening } \\
\text { without clear mass }\end{array}$ \\
\hline mrTRG 3 & $\begin{array}{l}\text { Moderate } \\
\text { response }\end{array}$ & $\begin{array}{l}>50 \% \text { fibrosis/mucin and visible } \\
\text { tumor with intermediate SI }\end{array}$ & $\begin{array}{l}>50 \% \text { fibrosis/mucin and visible } \\
\text { tumor with intermediate SI }\end{array}$ & \\
\hline mrTRG 4 & $\begin{array}{l}\text { Slight } \\
\text { response }\end{array}$ & $\begin{array}{l}\text { Little areas of fibrosis/mucin, } \\
\text { but mostly tumor }\end{array}$ & $\begin{array}{l}\text { Little areas of fibrosis/mucin, } \\
\text { but mostly tumor }\end{array}$ & $\begin{array}{l}\text { Residual mass (and/or } \\
\text { focal high SI on DWI) }\end{array}$ \\
\hline mrTRG 5 & $\begin{array}{l}\text { No } \\
\text { response }\end{array}$ & $\begin{array}{l}\text { Intermediate SI, same appearances } \\
\text { as original tumor/tumor regrowth }\end{array}$ & $\begin{array}{l}\text { Intermediate SI, same appearances } \\
\text { as original tumor/tumor regrowth }\end{array}$ & \\
\hline
\end{tabular}

DWI = diffusion-weighted imaging, ESGAR = European Society of Gastrointestinal and Abdominal Radiology, MERCURY = Magnetic Resonance Imaging and Rectal Cancer European Equivalence Study, mrTRG = MRI-based tumor regression grade, SI = signal intensity 

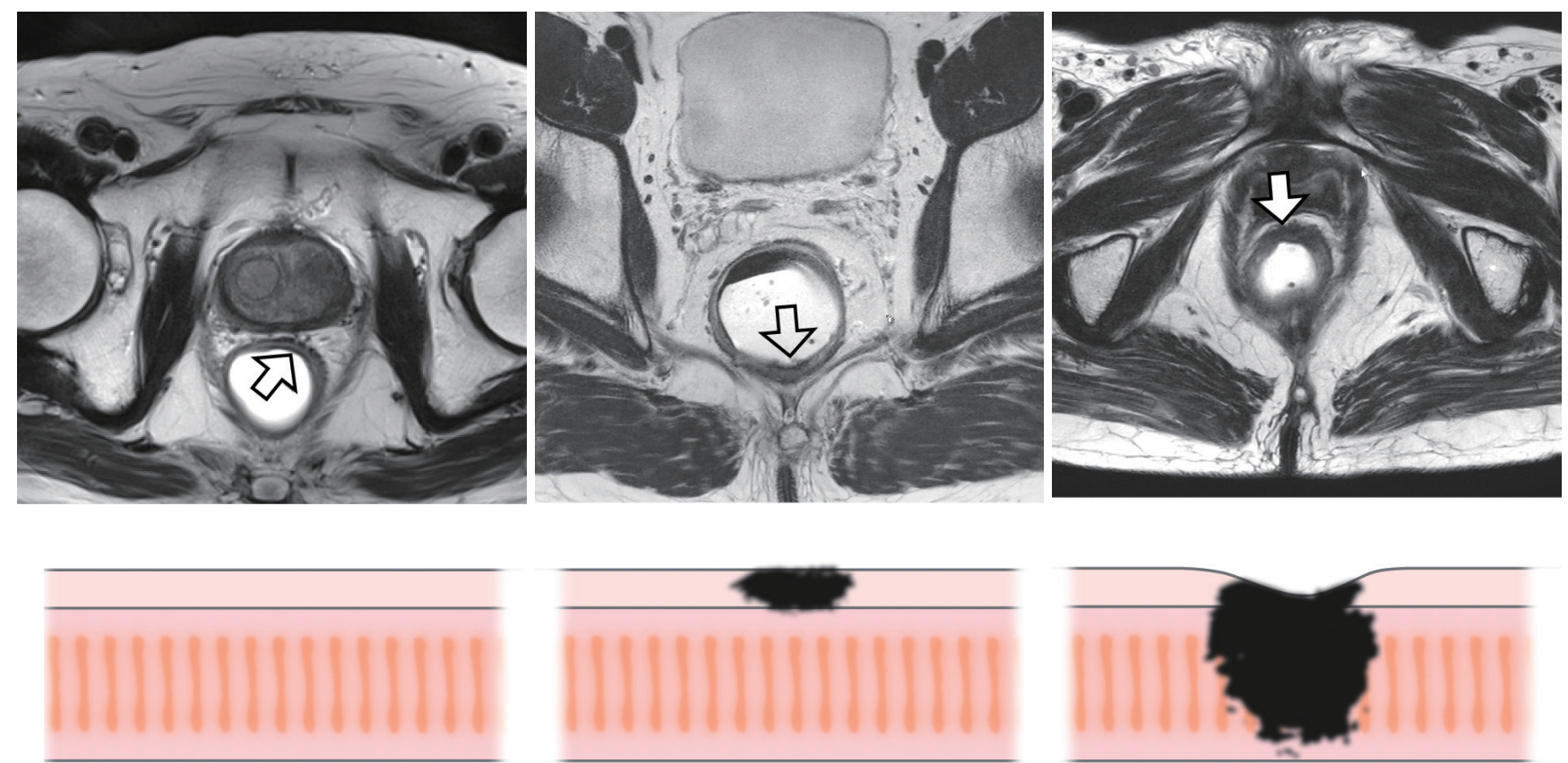

A
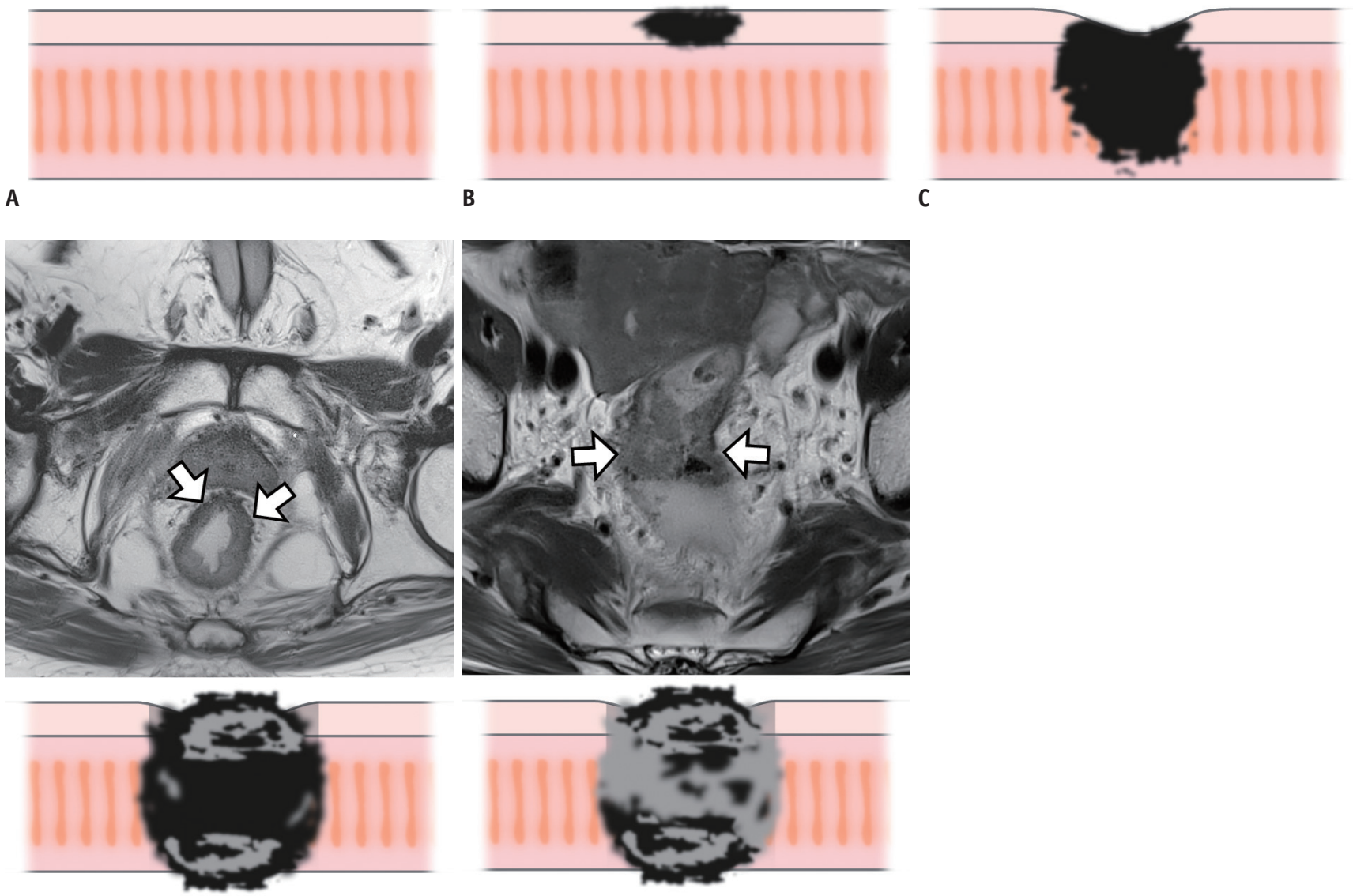

D

E

Fig. 5. Post-CRT mrTRG system.

A. ESGAR-based complete response: completely normalized rectal wall in initial tumor site (arrow). B. mrTRG 1: linear/crescentic scar in mucosa/ submucosal layer (arrow) which corresponds to ESGAR-based fibrotic wall thickening without clear mass. C. mrTRG 2: dense fibrotic transmural mass without intermediate tumor SI (arrow) which also corresponds to ESGAR-based fibrotic wall thickening without clear mass. D. mrTRG 3: more than $50 \%$ of areas with fibrosis and visible intermediate tumor (arrows) which corresponds to residual mass on ESGAR-based grading. E. mrTRG 4: few areas with fibrosis and mostly intermediate tumor SI (arrows) which also corresponds to residual mass on ESGAR-based grading. ESGAR = European Society of Gastrointestinal and Abdominal Radiology, mrTRG = MRI-based tumor regression grade

reports suggest only mrTRG 1 and 2 as good responders or candidates for organ-preserving treatments $(26,27)$.

Different from a 5-point mrTRG scoring system, ESGAR consensus meetings have suggested a three-stage classification: completely normalized wall, fibrotic wall thickening only, and residual mass. They proposed that an identification of a normalized two-layered wall after CRT is suggestive of CR on T2-weighted imaging (Fig. 5A) and 
fibrotic residue without an isointense mass may indicate CR or near-CR (Fig. 5B, C). However, the item related to a normalization of the rectal wall is not included in mrTRG scoring system proposed by MERCURY group. Therefore, the actual threshold for defining radiologic CR is somewhat different between the two scoring systems. Considering that complete normalization of the rectal wall after CRT is extremely rare in clinical practice, ESGAR group seems to take a stricter position in defining radiologic $C R$.

Local tumor status can also be assessed by post-treatment T staging (ymrT). On post-CRT MRI, yT stage can be defined as yT1-2, yT3, and yT4. yT3 can be further divided into yT3a or $3 b$ with $\leq 5 \mathrm{~mm}$ extramural tumor depth and $\mathrm{y} T 3 \mathrm{c}$ or $3 \mathrm{~d}$ with $>5 \mathrm{~mm}$ extramural tumor depth. yT4 is designated when the tumor invades into the visceral peritoneum or adjacent organs/structures (Fig. 6). Reported overall sensitivity of T2-weighted imaging in a meta-analysis is unsatisfactory (approximately 50\%) (28). Overstaging is the main source of staging error because CRT-induced fibrotic change at the interface between the tumor and the mesorectal fat can usually be mis-interpreted as viable tumor. The meta-analysis also revealed that restaging with diffusion-weighted imaging (DWI) demonstrated an improved mean sensitivity without a decrease of specificity. Therefore, DWI may be the solution for overcoming such overstaging issue (Fig. 7).

For tumors located in the far distal rectum, it is important to evaluate the relationship between the tumor

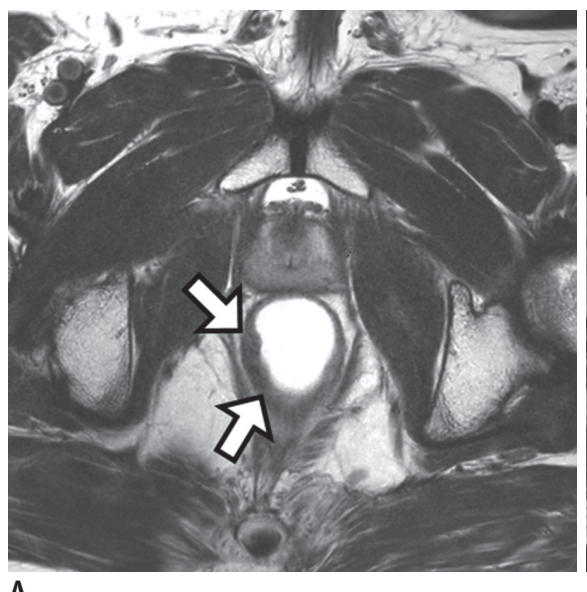

A

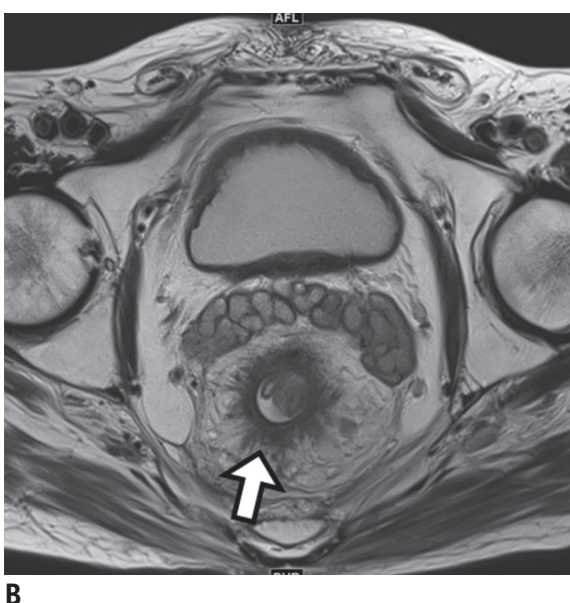

B

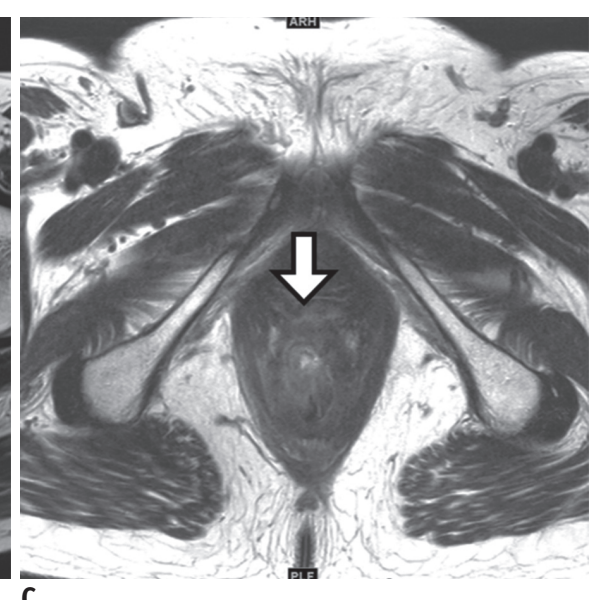

$\bar{C}$

Fig. 6. MRI-based T staging after CRT (ymrT) for rectal cancer.

A. Post-CRT MRI demonstrates localized transmural rectal wall thickening (ymrT1 or 2) with smooth outer margin (arrows). ypT2 was pathologically confirmed (not shown). B. Post-CRT MRI demonstrates circumferential mass (arrow) with irregular outer margin (ymrT3) within mesorectum. ypT3 was identified on histopathology (not shown). C. Post-CRT MRI reveals annular mass in lower rectum with posterior vagina infiltration (arrow) by tumor with intermediate SI (ymrT4b). After surgery, ypT4b was confirmed (not shown).

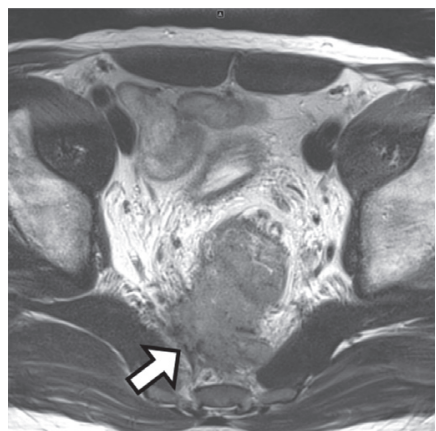

A
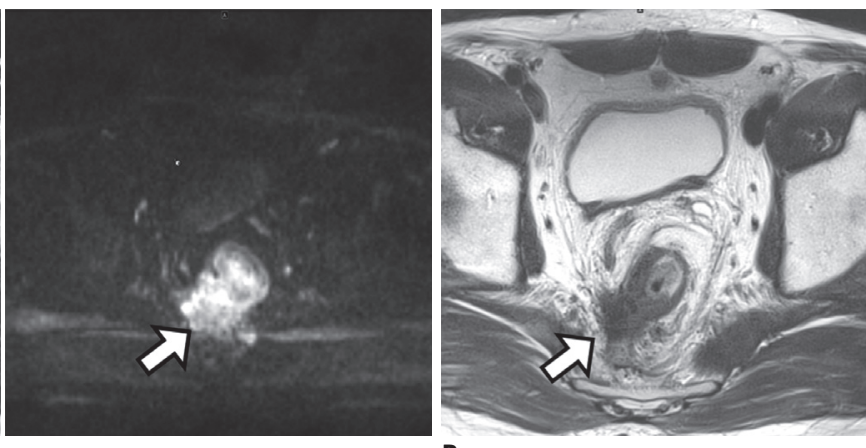

B

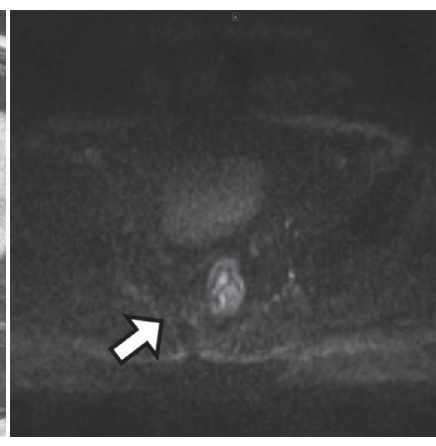

Fig. 7. Limitation of qualitative evaluation based on SI of anatomical T2-weighted imaging for tumor response evaluation.

A. Baseline T2-weighted image (left) shows bulky mass with MRF involvement at 8 o'clock direction (arrow). DWI (right) demonstrates strong diffusion restriction within whole tumor area (arrow). B. On post-CRT T2-weighted image (left), intermixed SI of tumor and fibrosis at initial tumor site (arrow) is noted. Therefore, mrTRG 3 was reported. CRM involvement by treated tumor is still suspected at same direction (arrow) because CRM threatening is determined by whole treated tumor including fibrotic component on post-CRT MRI interpretation. On DWI (right), however, no diffusion restriction is seen in entire tumor area (arrow). Pathologic complete response was confirmed after TME (not shown). CRM = circumferential resection margin, DWI $=$ diffusion-weighted imaging, $\mathrm{MRF}=$ mesorectal fascia, $\mathrm{TME}=$ total mesorectal excision 
and anal sphincter complex, particularly when deciding whether sphincter-saving surgery is possible or not (20). In such cases, a precise description regarding the relevant anatomical relationships may provide more valuable information than ymrT stage alone. Following findings are usually recommended to be described for lower tumors near the anal sphincter complex: 1) distal margin of the tumor whether it is located at above or below from the line $1 \mathrm{~cm}$ above the anorectal junction, 2) sphincter involvement by the tumor whether the tumor invades only to the internal sphincter muscle or extends to the intersphincteric fat plane/external sphincter muscle, and 3) involvement of levator ani muscle. The line $1 \mathrm{~cm}$ above the anorectal junction (black line in Fig. 3A) is approximately identical to an upper margin of the intersphincteric plane where is vulnerable to levator threatening $(9,10)$. Therefore, it is important to report the relationship between this line and distal margin of the tumor. For lower rectal cancers that threaten the levator ani muscle or involve the intersphincteric plane/external sphincter muscle, sphinctersaving surgery is not feasible. However, if the tumor shows a favorable response such as mrTRG 1 or 2 after CRT and no extramural tumor spread such as nodal metastasis is present on post-CRT MRI, organ-preserving surgery such as intersphincteric resection or TAE may be considered (26).

\section{Circumferential Resection Margin Involvement}

Pathologic circumferential resection margin (pCRM) is one of the key components for the determination of local tumor recurrence in patients who undergo TME with or without preoperative CRT $(25,29)$. Prospective trials have reported a local recurrence rate of $25-26 \%$ after CRT in patients with pCRM involvement $(25,30)$. The definition of positive PCRM in most studies is the presence of tumor cells within $1 \mathrm{~mm}$ from resection margin (31). On baseline MRI, when the shortest distance between the tumor and MRF is $\leq 1-2 \mathrm{~mm}$, potential CRM is considered to be threatened or involved. However, an interpretation of MRF involvement after CRT is problematic because hypointense fibrotic change frequently remains at the initial tumor area (Fig. 7). Such limitations result in a low PPV (44-57\%) and high negative predictive value (NPV) (91-100\%) of post-CRT MRI for positive pCRM $(16,32,33)$ with an area under the receiver operating characteristic curve (AUC) of $0.73-0.89(16,34,35)$. Although high NPV of post-CRT MRI may guarantee the oncologic safety after TME, low PPV prevents the decision for performing more aggressive or additional treatments. To overcome this issue, a few groups have suggested specific morphologic patterns on MRI for determining MRF involvement after CRT in addition to applying the distance from MRF (1-2 mm) $(16,34)$. Specifically, if MRF infiltration/penetration by fibrotic or tumor tissue is present along with fibrotic thickening of MRF itself, a higher risk of pCRM involvement after CRT is suggested compared to MRF threatening only by spiculated fibrotic change or treated tumor without thickening of MRF itself (Fig. 8). Some investigators have also reported the potential value of DWI to predict tumor clearance at the MRF after CRT (36). In this study, the PPV for MRF involvement was significantly higher with combined T2weighted imaging and DWI (82-91\%) than with T2weighted imaging alone $(30-45 \%)(p \leq 0.025)$ (36). If high-risk patients with positive $\mathrm{pCRM}$ even after CRT can be accurately selected, an evidence for introducing additional treatment options such as extramesorectal excision or multivisceral resection, boost radiotherapy at the site of MRF invasion, or upfront chemotherapy may be provided in these highly selected patients.

\section{Lymph Node Involvement}

Considering local excision may be a suitable option for patients with good tumor response to CRT without residual positive lymph nodes, radiologic nodal restaging after CRT is crucial for the assessment of these patients. MRI criteria used to determine lymph node involvement may differ between before and after CRT. Before CRT, morphologic findings such as border irregularities and heterogeneous SIs are better predictors than the size of lymph nodes (37). On the other hand, after CRT, these morphologic criteria were found to be unreliable predictors for determining malignant nodes $(38,39)$. Moreover, decreased nodal size (e.g., $<3 \mathrm{~mm}$ ) after CRT can limit the evaluation of these morphological features; thus the size criteria (e.g., a short axis $>5 \mathrm{~mm}$ ) may be more reliable.

Previous studies have indicated that size measurement after CRT shows better performance with an AUC of $0.85-$ 0.90 than primary staging $(A U C=0.75)(40,41)$. A possible explanation for such better performance of size criteria after CRT is that the size and number of lymph nodes harvested after CRT are reduced $(42,43)$, decreasing the potential for interpretation errors in the remaining small lymph nodes. On histology, many lymph nodes after CRT reveal fibrous capsular thickening and sclerosis within the medulla (44). In addition, radiologists should be aware that a decrease in 

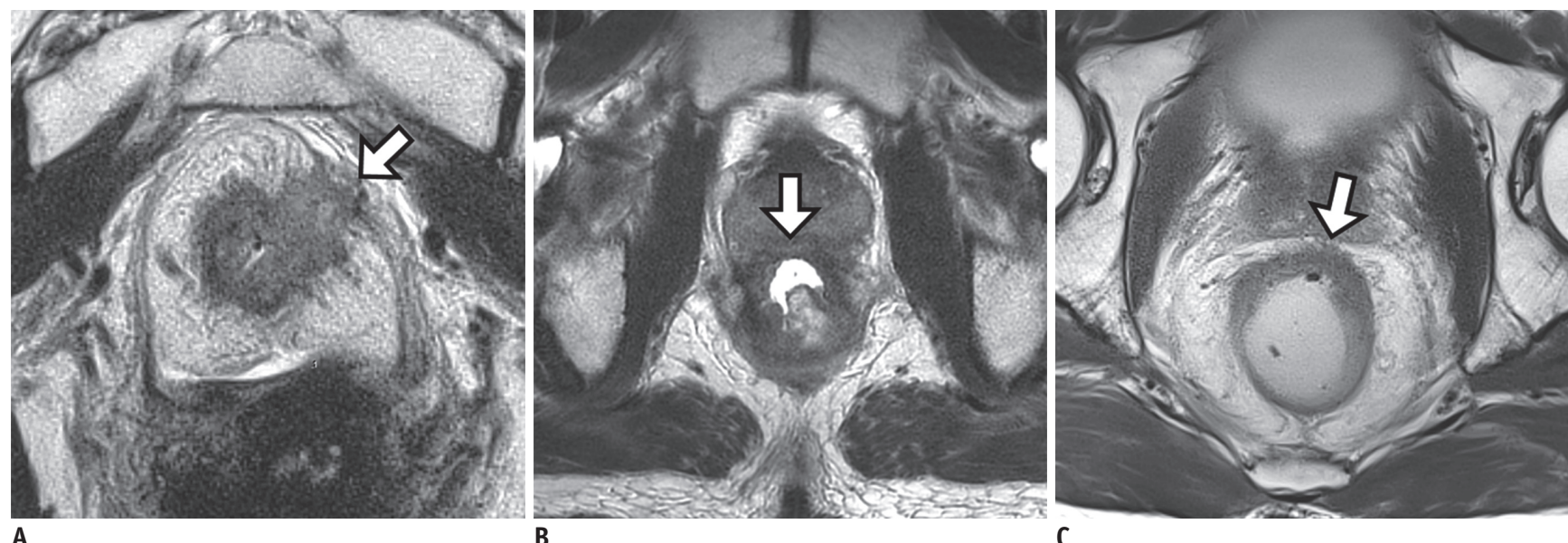

Fig. 8. Tumor penetration through MRF with MRF thickening which suggests high risk for positive pCRM even after completion of CRT.

A. Post-CRT MRI shows circumferential mass with intermediate SI. Tumor penetration to MRF with MRF thickening (arrow) is noted. Pathologic result after TME was positive pCRM (not shown). B. In another patient, post-CRT MRI still demonstrates tumor infiltration anteriorly into prostatic gland (arrow). After APE with partial prostatectomy, pCRM was found to be positive. C. On post-CRT MRI in another patient, tumor with anterior MRF threatening by spicules of treated tumor (arrow) is noted. However, MRF thickening or penetration is not demonstrated (arrow). Pathologic result after TME was negative pCRM (not shown).

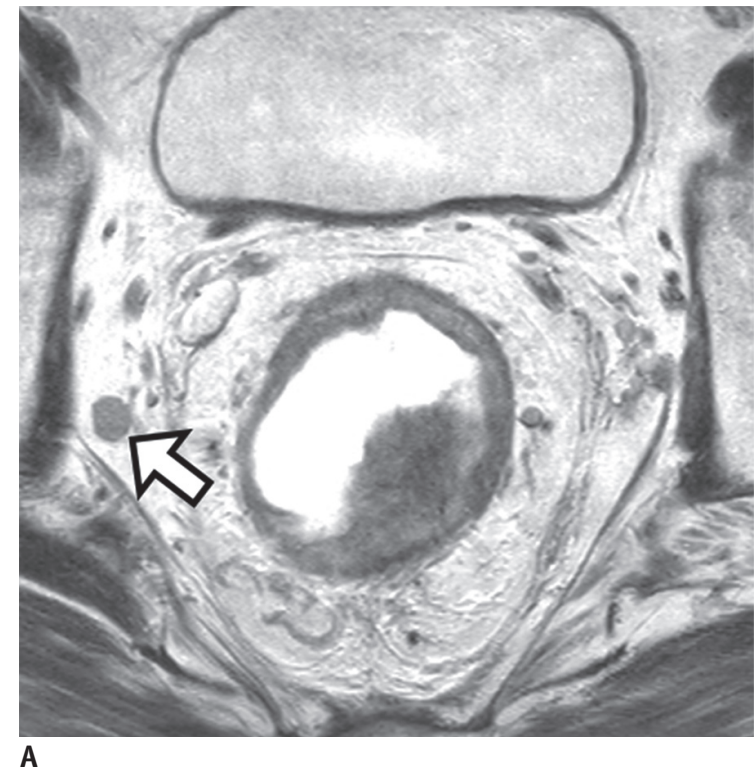

A

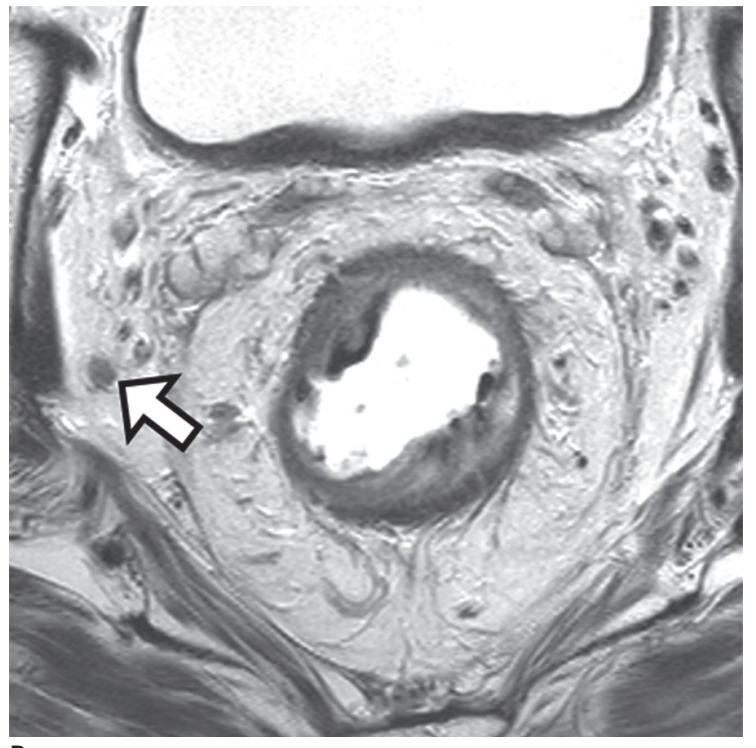

B

Fig. 9. Tumor response of lymph node after CRT.

A. On baseline MRI, suspicious metastatic node with short axis diameter of $9 \mathrm{~mm}$ is seen at right lateral pelvic side wall (arrow). B. Post-CRT MRI reveals interval decrease of node (arrow) and size is less than $5 \mathrm{~mm}$. Lymph node dissection at right lateral pelvic side wall was performed. Viable tumor cells were not found in dissected lymph nodes (not shown). However, pathologic nodal status after CRT still remains uncertain because both normal and metastatic lymph nodes show interval decrease in size after CRT.

size of nodes after CRT cannot always guarantee malignant nodes because benign as well as malignant nodes can show decrease in size after CRT $(43,44)$ (Fig. 9).

In lateral pelvic lymph nodes, the same size criteria (a short axis $>5 \mathrm{~mm}$ ) can be used to reliably identify malignant nodes after CRT $(45,46)$ (Fig. 10). Persistently enlarged lateral pelvic nodes $>5 \mathrm{~mm}$ on post-CRT MRI are significantly associated with residual nodal metastasis and the size of lateral pelvic node on post-CRT MRI is an independent predictor for lymph node metastasis (46). Therefore, the decision of selective lateral pelvic node dissection should be made on the basis of nodal response to CRT (e.g., via size of lateral pelvic node on post-CRT MRI) (Table 1) $(45,46)$. For patients with responsive lateral 


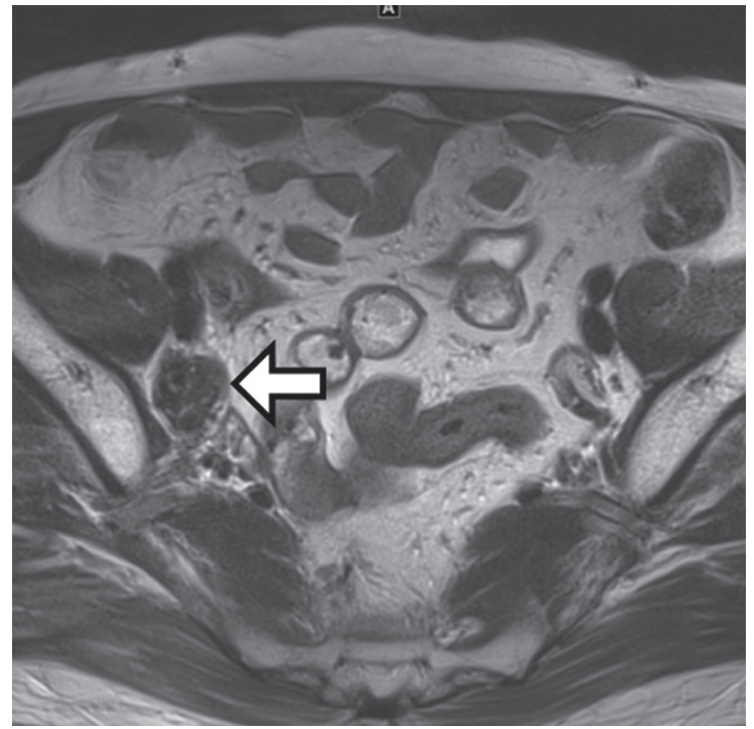

A

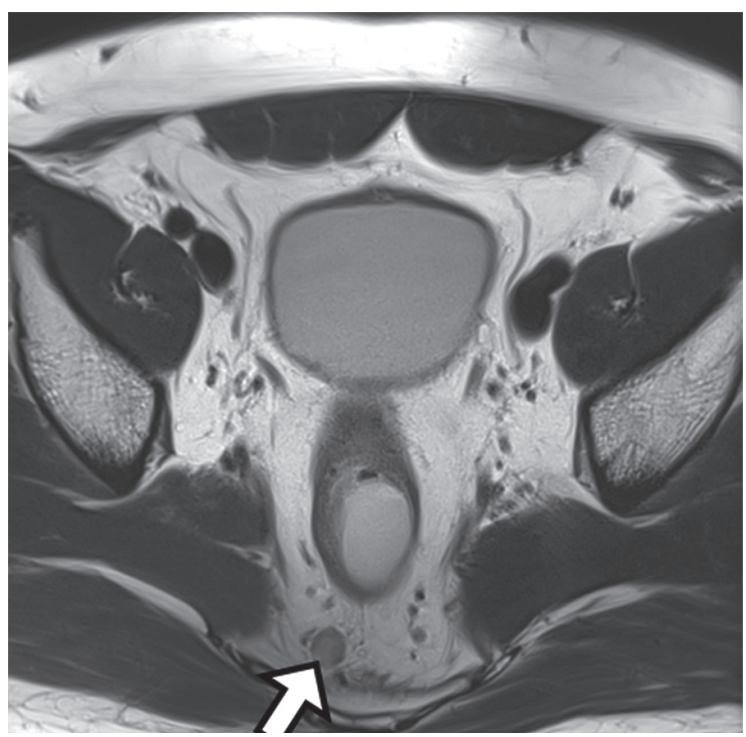

B

Fig. 10. Metastatic lymph nodes after CRT.

A. Post-CRT MRI reveals persistently enlarged lymph node (arrow) with short axis diameter of $16 \mathrm{~mm}$ in right obturator area. Selective lymph node dissection at right pelvic side wall is performed and lymph node metastasis is pathologically confirmed (not shown). B. In another patient, post-CRT MRI demonstrates persistently enlarged node (arrow) with short axis diameter of $7.5 \mathrm{~mm}$ within mesorectum. After TME, metastasis is also identified within lymph node (not shown).

pelvic nodes (short axis $<5 \mathrm{~mm}$ ) after CRT, lateral pelvic node dissection may not be necessary although there has been still a debate on this issue.

\section{Extramural Venous Invasion}

EMVI is pathologically defined when tumor cells invading the veins beyond the outer walls of the muscularis propria are present (47). After CRT, EMVI can be assessed either by histopathology (ypEMVI) or by MRI (ymrEMVI). ymrEMVI is defined as positive when minimal or less than $25 \%$ fibrotic change is detected on treated tumor component within the extramural venous structure (Fig. 11) $(48,49)$.

Lee et al. (48) reported that the diagnostic performance of MRI for determining EMVI after CRT was comparable to that on pre-CRT MRI (AUC, 0.830 versus $0.778, p=$ 0.076) when using the same scoring system for mrEMVI. According to another study by Chand et al. (47), positive EMVI after CRT was more frequently detected on MRI than on histopathology (53\% versus 19\%) and both ymrEMVI (hazard ratio 1.97) and ypEMVI (hazard ratio 2.39) were significant predictors for decreased survival. Considering the advantages of MRI such as multiplanar imaging capability and visualization of the entire rectum (50), radiationinduced fibrosis and destruction of venous endothelium after CRT may be responsible for a higher false-negative rate of EMVI on histopathology (51). As persistent ymrEMVI after CRT may increase the risks of metastatic disease, it is an independent negative prognostic marker for diseasefree survival and implicit potential benefits of adjuvant chemotherapy (47).

\section{Determining Complete Response on Post- Chemoradiotherapy MRI}

Most LARCs show variable tumor response, including pCR in up to $33 \%$ of patients $(21,52)$. Patients who achieved CR after CRT may potentially achieve excellent local tumor control and superior quality of life with organpreservation strategies such as local excision or even a watch-and-wait approach (53-55). If patients who have achieved pCR after CRT can be accurately identified before surgery, organ-preservation treatment strategies might be considered as an option in those selective patients. By avoiding radical surgery in these patients, the need for a permanent stoma may be avoided (53-55). However, if an inaccurate assessment for $\mathrm{PCR}$ status was made, the patients may lose the chance of standard radical TME surgery. Resultantly, oncologic safety of those patients may be severely jeopardized. Consequently, an increasing interest in organ-saving treatments can be met only if a reliable method to identify $\mathrm{PCR}$ is developed $(56,57)$. In reality, however, an accurate identification and prediction 


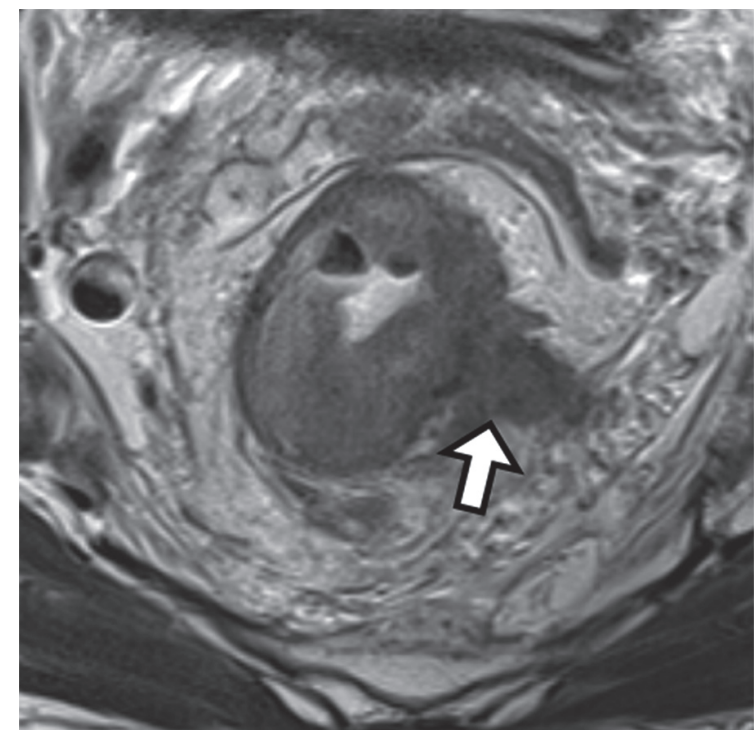

A

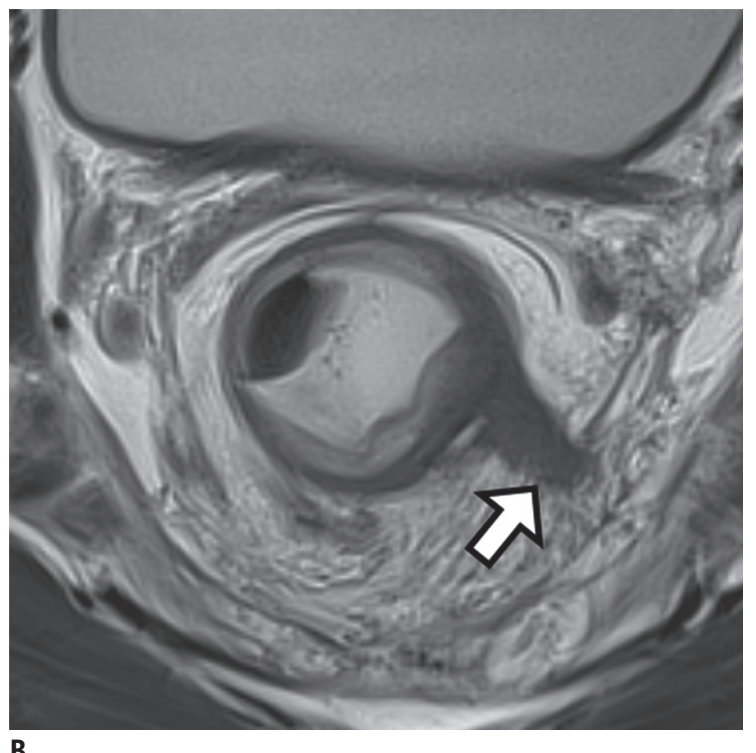

B

Fig. 11. Positive EMVI on post-CRT MRI (ymrEMVI).

A. Baseline MRI shows positive mrEMVI of grade 4 which indicates irregular vessels replaced by soft tissue with intermediate SI (arrow). B. PostCRT MRI still depicts positive ymrEMVI with minimal fibrotic change showing low SI (at least less than 25\%) although prominent shrinkage is noted at primary rectal mass. EMVI $=$ extramural venous invasion

Table 3. MRI Findings Indicating $p C R$

\begin{tabular}{ll}
\hline \multicolumn{1}{c}{ Imaging Modality } & Imaging Findings Indicating $\mathrm{pCR}$ \\
\hline T2-weighted imaging & - Complete normalization of rectal wall \\
DWI & - Dense fibrotic lesion with low SI similar to pelvic muscle without intermediate tumor SI \\
T2 SI or DWI selective volumetry & No evidence of diffusion restriction within tumor \\
\hline
\end{tabular}

${ }^{*}$ Cut-off values for predicting $\mathrm{pCR}$ have not yet been established. $\mathrm{pCR}=$ pathologic complete remission

of pCR is still challenging, as a reliable and non-invasive method is not yet fully available (58). Limited ability of post-CRT MRI to predict $\mathrm{pCR}$ also leaves a gap in the clinical process of determining a reasonable treatment strategy. The ideal balance between the sensitivity and specificity of MRI for the prediction of pCR is an ongoing issue of debate. Considering that a false-positive prediction of $\mathrm{pCR}$ can lead to an inappropriate use of organ-preserving treatments, potentially resulting in devastating outcomes, a conservative decision for CCR should be made to maximize the PPV of post-CRT MRI for the prediction of $p C R$ when the results are equivocal.

Recently, a large-scale registry study investigated the clinical outcomes of patients with $\mathrm{CCR}$, reporting a 2-year cumulative local regrowth rate of $25.2 \%$ (59). Among cCR patients with local regrowth, $97 \%$ of local regrowth was detected at the bowel wall while distant metastasis occurred in $8 \%$ of cCR patients. Based on this result, unsalvageable disease is rare even in CCR patients with later tumor regrowth (59). Therefore, this data support that a watch-and-wait strategy or deferred curative surgery can be performed as reasonable treatment options for CCR patients if an adequate clinical surveillance is guaranteed.

\section{T2-Weighted Imaging}

Pathologic CR can be theoretically achieved in patients with mrTRG 1 and 2 (MERCURY classification) or complete normalization and fibrotic tissue (ESGAR classification) (Table 3). Clinical CR on T2-weighted MRI can be diagnosed when intermediate tumor signal disappears with or without fibrotic residues (Fig. 12). However, characterizing residual tumor with MRI is challenging because a distinction between fibrosis with and without residual tumors is difficult. Although a signal drop of the tumor on T2weighted image is widely accepted as a hallmark of CRTinduced fibrosis, fibrosis frequently intermixes with viable tumor tissues and distinguishing these two components is often a difficult task (23). Furthermore, hyperintense 


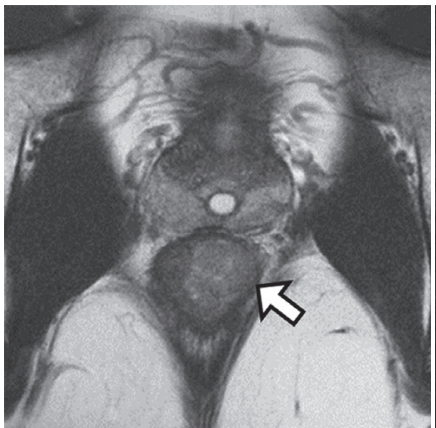

A

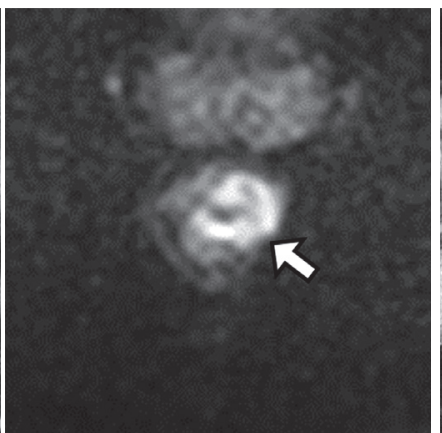

B
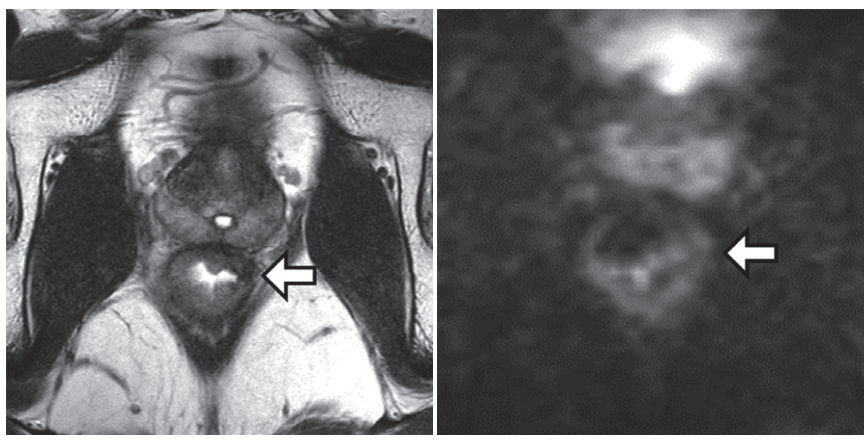

Fig. 12. Typical MRI findings of pathologic complete response after CRT.

A. Baseline MRI (left) shows fungating tumor (arrow) with intermediate SI in lower rectum. DWI (right) demonstrates prominent diffusion restriction at tumor (arrow). B. Post-CRT MRI (left) reveals transmural fibrosis with dark SI in left lateral wall of rectum (arrow). There is no demonstrable residual tumor with intermediate SI. DWI (right) shows no evidence of diffusion restriction at corresponding tumor site.

mucin on T2-weighted image makes the prediction of residual tumor viability more challenging (60). Intermingled tumor cells with fibrosis and the presence of mucin may be responsible for low level of agreement between mrTRG and PTRG, rendering mrTRG as an inappropriate surrogate for the prediction of pTRG (61). Indeed, the reported sensitivity and specificity for diagnosing $\mathrm{pCR}$ are $74.4 \%$ and $62.8 \%$, respectively even in patients showing good response on post-CRT MRI (mrTRG 1 or 2) (61).

\section{MR Tumor Volumetry}

There have been several reports regarding the usefulness of MR volumetry for the prediction of tumor response after CRT $(62,63)$. In those studies, investigators used both quantitative SI and volume of the tumor in order to selectively measure the volume of viable tumor tissues on post-CRT MRI. They performed SI-selective volumetry with an exclusion of non-viable tissue components (fibrosis with low SI and mucin with high SI) using an intensity threshold. For example, pelvic muscle was used as an internal reference to define a threshold of fibrotic tissue. This approach seemed to improve the diagnostic performance of MRI for $\mathrm{pCR}$ assessment, compared with simple qualitative assessment or whole tumor volumetry $(62,63)$.

\section{Diffusion-Weighted Imaging}

Recently, DWI has been increasingly used and is routinely included in rectal MRI protocols (20). A few studies have reported encouraging results on DWI, describing its usefulness in aiding conventional MRI to improve the diagnostic accuracy for predicting pCR after CRT (Figs. 7, 12) $(64,65)$.

Even though DWI offers several benefits in various applications, it also has multiple possible shortcomings. For example, manual drawing of ROIs onto the tumor for quantitative or qualitative assessment may result in interobserver variation. Furthermore, image distortion due to artifacts is common on DWI, particularly around airtissue interfaces. During the restaging of tumor, radiologists tend to overestimate the presence of a tumor on T2weighted image alone. According to previous studies, such overestimation can be reduced when DWI was added on T2weighted image (64-66). In real clinical practice, however, such corrective effect of DWI may not be encountered because most conservative radiologists would not change their decision to $\mathrm{CCR}$ even in patients with no diffusion restriction on DWI if when residual tumor signal is present on T2-weighted imaging.

According to a previous study, DWI volumetry without T2-weighted imaging showed highest AUC $(>0.90)$ for the assessment of pCR. This observation might be due to the fact that the bias related to T2-weighted imaging can be eliminated in this study (66). Another subsequent biinstitutional independent validation study proved that the previously established cutoff value for DWI volumetry could be used to assess pCR after CRT accurately (15). Therefore, DWI volumetry may have some potential to overcome intrinsic limitations of qualitative DWI-related artifact or radiologists' conservative tendency.

\section{Combined Use of MRI and Colonoscopy}

In the past, endoscopy and digital rectal exams served as the main tools for the assessment of treatment response $(15,67)$. Continuous decrease in tumor size and the disappearance of tumor tissues along with mucosal healing, were regarded as clinical signs of CR on endoscopy 
(57). However, a major limitation of these techniques is that transmural or extraluminal tumor extent as well as mesorectum cannot be examined (57). Because residual tumors can present in any layer of the rectal wall regardless of tumor stage $(57,68,69)$, MRI can reveal such residual tumors beneath the mucosa, which can be inaccessible via colonoscopy.

Endoscopy and MRI may play a complementary role in rectal cancer restaging. Maas et al. (57) reported that the combined use of MRI and endoscopy resulted in a high PPV (98\%). Although an improved PPV maximizes oncologic safety, it also decreases the opportunity of organ-preserving treatment in pCR patients with equivocal findings (57). However, we believe that such a conservative approach may be reasonable considering the limitation of imaging tools for predicting $\mathrm{PCR}$ after CRT and oncologic safety.

\section{Selection of Candidates for Local Excision after Chemoradiotherapy}

Considering the limitations of MRI or endoscopy in predicting $\mathrm{pCR}$, local excision may be a more go-ahead approach. Indeed, there have been conflicting arguments for the indications for local excision. Some reports have demonstrated acceptable oncologic outcomes after local excision for ypT2 lesions $(42,54,70)$ while lymph node metastasis was reported in up to $20 \%$ of ypT2 lesions (38, 71, 72).

The most important issue in selecting patients for local excision is the guarantee of pNO before an organpreserving treatment because lymph node removal is not performed and any residual viable tumor deposit may be left behind after local excision (42). Indeed, the risk for tumor recurrence after local excision has been described to parallel the risk of lymph node metastasis (2.0-9.1\% for ypT0, $15.0-17.1 \%$ for ypT1, and $17.0-20.8 \%$ for ypT2) (38, $71,72)$. Therefore, an acceptable NPV for the presence of metastatic nodes is crucial for planning organ-preserving treatment. Even though the use of size criteria on post-CRT MRI shows a relatively high NPV $(78-96 \%)(38,70)$, such imperfect NPV does not entirely guarantee the oncologic safety after local excision. Therefore, the risk of local tumor recurrence due to residual metastatic lymph nodes should be compensated by strict MRI follow-up so as to facilitate early detection during the time window in which curative radical resection can still be performed (42).

\section{SUMMARY}

Along with significant improvement in terms of treatment options and imaging tools, there is an increasing demand for reliable risk stratification after CRT for personalized tailored treatments in patients with LARC. Radiologists should be familiar with new surgical approaches. With the knowledge of oncologic concepts specific to rectal cancer management, radiologists should provide critical information during post-CRT MRI interpretation such as mrTRG, ycTN staging, relationship with anal sphincter complex, CRM status, and ymrEMVI. Post-CRT MRI may play a prominent role in the decision-making process for rectal cancer management. However, because current MRI techniques have several limitations for assessing treatment response after CRT, tumor response evaluation on postCRT MRI should be reliably or safely determined with an aid of DWI, MRI volumetry, and complementary endoscopy, balancing between oncologic safety and quality of life for patients.

\section{Conflicts of Interest}

The authors have no potential conflicts of interest to disclose.

ORCID iDs

Joon Seok Lim

https://orcid.org/0000-0002-0334-5042

Nieun Seo

https://orcid.org/0000-0001-8745-6454

Honsoul Kim

https://orcid.org/0000-0002-2367-234X

Min Soo Cho

https://orcid.org/0000-0002-7310-4893

\section{REFERENCES}

1. KSAR Study Group for Rectal Cancer. Essential items for structured reporting of rectal cancer MRI: 2016 consensus recommendation from the Korean Society of Abdominal Radiology. Korean J Radiol 2017;18:132-151

2. Costa-Silva L, Brown G. Magnetic resonance imaging of rectal cancer. Magn Reson Imaging Clin N Am 2013;21:385-408

3. García-Aguilar J, Hernandez de Anda E, Sirivongs P, Lee $\mathrm{SH}$, Madoff RD, Rothenberger DA. A pathologic complete response to preoperative chemoradiation is associated with lower local recurrence and improved survival in rectal cancer patients treated by mesorectal excision. Dis Colon Rectum 
2003;46:298-304

4. Glynne-Jones R. Rectal cancer--the times they are a-changing. Lancet Oncol 2012;13:651-653

5. Tepper JE, O'Neil BH. Minimizing therapy and maximizing outcomes in rectal cancer. J Clin Oncol 2011;29:4604-4606

6. Heald RJ, Ryall RD. Recurrence and survival after total mesorectal excision for rectal cancer. Lancet 1986;1:14791482

7. Kim NK, Sugihara K, Liang JT. Surgical treatment of colorectal cancer: Asian perspectives on optimization and standardization. Singapore: Springer Singapore, 2018

8. Kuvshinoff B, Maghfoor I, Miedema B, Bryer M, Westgate S, Wilkes J, et al. Distal margin requirements after preoperative chemoradiotherapy for distal rectal carcinomas: are $<$ or $=1$ cm distal margins sufficient? Ann Surg Oncol 2001;8:163-169

9. Battersby NJ, How P, Moran B, Stelzner S, West NP, Branagan G, et al.; MERCURY II Study Group. Prospective validation of a low rectal cancer magnetic resonance imaging staging system and development of a local recurrence risk stratification model: the MERCURY II study. Ann Surg 2016;263:751-760

10. Shihab OC, Heald RJ, Rullier E, Brown G, Holm T, Quirke P, et al. Defining the surgical planes on MRI improves surgery for cancer of the low rectum. Lancet Oncol 2009;10:1207-1211

11. Nagtegaal ID, van de Velde CJ, Marijnen CA, van Krieken JH, Quirke P; Dutch Colorectal Cancer Group; Pathology Review Committee. Low rectal cancer: a call for a change of approach in abdominoperineal resection. J Clin Oncol 2005;23:92579264

12. Yu HC, Peng H, He XS, Zhao RS. Comparison of short- and long-term outcomes after extralevator abdominoperineal excision and standard abdominoperineal excision for rectal cancer: a systematic review and meta-analysis. Int J Colorectal Dis 2014;29:183-191

13. Yamada K, Ishizawa T, Niwa K, Chuman Y, Akiba S, Aikou T. Patterns of pelvic invasion are prognostic in the treatment of locally recurrent rectal cancer. Br J Surg 2001;88:988-993

14. Smith JJ, Garcia-Aguilar J. Advances and challenges in treatment of locally advanced rectal cancer. J Clin Oncol 2015;33:1797-1808

15. Lambregts DM, Rao SX, Sassen S, Martens MH, Heijnen LA, Buijsen J, et al. MRI and diffusion-weighted MRI volumetry for identification of complete tumor responders after preoperative chemoradiotherapy in patients with rectal cancer: a Bi-institutional validation study. Ann Surg 2015;262:1034-1039

16. Vliegen RF, Beets GL, Lammering G, Dresen RC, Rutten HJ, Kessels AG, et al. Mesorectal fascia invasion after neoadjuvant chemotherapy and radiation therapy for locally advanced rectal cancer: accuracy of MR imaging for prediction. Radiology 2008;246:454-462

17. Cummings BJ, Rider WD, Harwood AR, Keane TJ, Thomas GM. Radical external beam radiation therapy for adenocarcinoma of the rectum. Dis Colon Rectum 1983;26:30-36

18. Goodman KA. Timing is everything: what is the optimal duration after chemoradiation for surgery for rectal cancer? J Clin Oncol 2016;34:3724-3728

19. Lefevre JH, Mineur L, Kotti S, Rullier E, Rouanet P, de Chaisemartin C, et al. Effect of interval (7 or 11 weeks) between neoadjuvant radiochemotherapy and surgery on complete pathologic response in rectal cancer: a multicenter, randomized, controlled trial (GRECCAR-6). J Clin Oncol 2016;34:3773-3780

20. Beets-Tan RGH, Lambregts DMJ, Maas M, Bipat S, Barbaro B, Curvo-Semedo $L$, et al. Magnetic resonance imaging for clinical management of rectal cancer: updated recommendations from the 2016 European Society of Gastrointestinal and Abdominal Radiology (ESGAR) consensus meeting. Eur Radiol 2018;28:1465-1475

21. Patel UB, Brown G, Rutten H, West N, Sebag-Montefiore D, Glynne-Jones R, et al. Comparison of magnetic resonance imaging and histopathological response to chemoradiotherapy in locally advanced rectal cancer. Ann Surg Oncol 2012; 19:2842-2852

22. Bhoday J, Smith F, Siddiqui MR, Balyasnikova S, Swift RI, Perez $\mathrm{R}$, et al. Magnetic resonance tumor regression grade and residual mucosal abnormality as predictors for pathological complete response in rectal cancer postneoadjuvant chemoradiotherapy. Dis Colon Rectum 2016;59:925-933

23. Taylor FG, Swift RI, Blomqvist L, Brown G. A systematic approach to the interpretation of preoperative staging MRI for rectal cancer. AJR Am J Roentgenol 2008;191:1827-1835

24. Patel UB, Blomqvist LK, Taylor F, George C, Guthrie A, Bees N, et al. MRI after treatment of locally advanced rectal cancer: how to report tumor response--the MERCURY experience. AJR Am J Roentgenol 2012;199:W486-W495

25. Patel UB, Taylor F, Blomqvist L, George C, Evans H, Tekkis P, et al. Magnetic resonance imaging-detected tumor response for locally advanced rectal cancer predicts survival outcomes: MERCURY experience. J Clin Oncol 2011;29:3753-3760

26. Jang JK, Lee JL, Park SH, Park HJ, Park IJ, Kim JH, et al. Magnetic resonance tumour regression grade and pathological correlates in patients with rectal cancer. Br J Surg 2018;105:1671-1679

27. Battersby NJ, Dattani M, Rao S, Cunningham D, Tait D, Adams $R$, et al. A rectal cancer feasibility study with an embedded phase III trial design assessing magnetic resonance tumour regression grade (mrTRG) as a novel biomarker to stratify management by good and poor response to chemoradiotherapy (TRIGGER): study protocol for a randomised controlled trial. Trials 2017;18:394

28. van der Paardt MP, Zagers MB, Beets-Tan RG, Stoker J, Bipat $S$. Patients who undergo preoperative chemoradiotherapy for locally advanced rectal cancer restaged by using diagnostic MR imaging: a systematic review and meta-analysis. Radiology 2013;269:101-112

29. Nagtegaal ID, Quirke P. What is the role for the circumferential margin in the modern treatment of rectal cancer? J Clin Oncol 2008;26:303-312 
30. Sauer R, Liersch T, Merkel S, Fietkau R, Hohenberger W, Hess $C$, et al. Preoperative versus postoperative chemoradiotherapy for locally advanced rectal cancer: results of the German CAO/ ARO/AIO-94 randomized phase III trial after a median followup of 11 years. J Clin Oncol 2012;30:1926-1933

31. Adam IJ, Mohamdee M0, Martin IG, Scott N, Finan PJ, Johnston $D$, et al. Role of circumferential margin involvement in the local recurrence of rectal cancer. Lancet 1994;344:707711

32. MERCURY Study Group. Diagnostic accuracy of preoperative magnetic resonance imaging in predicting curative resection of rectal cancer: prospective observational study. BMJ 2006;333:779

33. Kulkarni T, Gollins S, Maw A, Hobson P, Byrne R, Widdowson $D$. Magnetic resonance imaging in rectal cancer downstaged using neoadjuvant chemoradiation: accuracy of prediction of tumour stage and circumferential resection margin status. Colorectal Dis 2008;10:479-489

34. Kim KH, Park MJ, Lim JS, Kim NK, Min BS, Ahn JB, et al. Circumferential resection margin positivity after preoperative chemoradiotherapy based on magnetic resonance imaging for locally advanced rectal cancer: implication of boost radiotherapy to the involved mesorectal fascia. Jpn J Clin Oncol 2016;46:316-322

35. Kim SH, Lee JM, Park HS, Eun HW, Han JK, Choi BI. Accuracy of MRI for predicting the circumferential resection margin, mesorectal fascia invasion, and tumor response to neoadjuvant chemoradiotherapy for locally advanced rectal cancer. J Magn Reson Imaging 2009;29:1093-1101

36. Park MJ, Kim SH, Lee SJ, Jang KM, Rhim H. Locally advanced rectal cancer: added value of diffusion-weighted MR imaging for predicting tumor clearance of the mesorectal fascia after neoadjuvant chemotherapy and radiation therapy. Radiology 2011;260:771-780

37. Brown G, Richards CJ, Bourne MW, Newcombe RG, Radcliffe AG, Dallimore NS, et al. Morphologic predictors of lymph node status in rectal cancer with use of high-spatial-resolution MR imaging with histopathologic comparison. Radiology 2003;227:371-377

38. Perez R0, Pereira DD, Proscurshim I, Gama-Rodrigues J, Rawet V, São Julião GP, et al. Lymph node size in rectal cancer following neoadjuvant chemoradiation--can we rely on radiologic nodal staging after chemoradiation? Dis Colon Rectum 2009;52:1278-1284

39. Lahaye MJ, Beets GL, Engelen SM, Kessels AG, de Bruïne AP, Kwee HW, et al. Locally advanced rectal cancer: MR imaging for restaging after neoadjuvant radiation therapy with concomitant chemotherapy. Part II. What are the criteria to predict involved lymph nodes? Radiology 2009;252:81-91

40. Kusters M, Marijnen CA, van de Velde CJ, Rutten HJ, Lahaye $\mathrm{MJ}$, Kim JH, et al. Patterns of local recurrence in rectal cancer; a study of the Dutch TME trial. Eur J Surg Oncol 2010;36:470-476

41. Lahaye MJ, Engelen SM, Kessels AG, de Bruïne AP, von
Meyenfeldt MF, van Engelshoven JM, et al. USPIO-enhanced MR imaging for nodal staging in patients with primary rectal cancer: predictive criteria. Radiology 2008;246:804-811

42. Engelen SM, Beets-Tan RG, Lahaye MJ, Lammering G, Jansen $\mathrm{RL}$, van Dam RM, et al. MRI after chemoradiotherapy of rectal cancer: a useful tool to select patients for local excision. Dis Colon Rectum 2010;53:979-986

43. Koh DM, Chau I, Tait D, Wotherspoon A, Cunningham D, Brown G. Evaluating mesorectal lymph nodes in rectal cancer before and after neoadjuvant chemoradiation using thinsection T2-weighted magnetic resonance imaging. Int $\mathrm{J}$ Radiat Oncol Biol Phys 2008;71:456-461

44. Prall F, Wöhlke M, Klautke G, Schiffmann L, Fietkau R, Barten $M$. Tumour regression and mesorectal lymph node changes after intensified neoadjuvant chemoradiation for carcinoma of the rectum. APMIS 2006;114:201-210

45. Akiyoshi T, Ueno M, Matsueda K, Konishi T, Fujimoto Y, Nagayama $S$, et al. Selective lateral pelvic lymph node dissection in patients with advanced low rectal cancer treated with preoperative chemoradiotherapy based on pretreatment imaging. Ann Surg Oncol 2014;21:189-196

46. Oh HK, Kang SB, Lee SM, Lee SY, Ihn MH, Kim DW, et al. Neoadjuvant chemoradiotherapy affects the indications for lateral pelvic node dissection in mid/low rectal cancer with clinically suspected lateral node involvement: a multicenter retrospective cohort study. Ann Surg Oncol 2014;21:22802287

47. Chand M, Evans J, Swift RI, Tekkis PP, West NP, Stamp G, et al. The prognostic significance of postchemoradiotherapy high-resolution MRI and histopathology detected extramural venous invasion in rectal cancer. Ann Surg 2015;261:473-479

48. Lee ES, Kim MJ, Park SC, Hur BY, Hyun JH, Chang HJ, et al. Magnetic resonance imaging-detected extramural venous invasion in rectal cancer before and after preoperative chemoradiotherapy: diagnostic performance and prognostic significance. Eur Radiol 2018;28:496-505

49. Chand M, Swift RI, Tekkis PP, Chau I, Brown G. Extramural venous invasion is a potential imaging predictive biomarker of neoadjuvant treatment in rectal cancer. $\mathrm{Br} \mathrm{J}$ Cancer 2014;110:19-25

50. Ale Ali H, Kirsch R, Razaz S, Jhaveri A, Thipphavong S, Kennedy ED, et al. Extramural venous invasion in rectal cancer: overview of imaging, histopathology, and clinical implications. Abdom Radiol (NY) 2019;44:1-10

51. Liang P, Nakada I, Hong JW, Tabuchi T, Motohashi G, Takemura A, et al. Prognostic significance of immunohistochemically detected blood and lymphatic vessel invasion in colorectal carcinoma: its impact on prognosis. Ann Surg Oncol 2007; 14:470-477

52. Francois Y, Nemoz CJ, Baulieux J, Vignal J, Grandjean $\mathrm{JP}$, Partensky $\mathrm{C}$, et al. Influence of the interval between preoperative radiation therapy and surgery on downstaging and on the rate of sphincter-sparing surgery for rectal cancer: the Lyon R90-01 randomized trial. J Clin Oncol 1999;17:2396- 
2402

53. Maas M, Nelemans PJ, Valentini V, Das P, Rödel C, Kuo LJ, et al. Long-term outcome in patients with a pathological complete response after chemoradiation for rectal cancer: a pooled analysis of individual patient data. Lancet Oncol 2010;11:835-844

54. Borschitz T, Wachtlin D, Möhler M, Schmidberger H, Junginger T. Neoadjuvant chemoradiation and local excision for T2-3 rectal cancer. Ann Surg Oncol 2008;15:712-720

55. Park IJ, Yu CS. Current issues in locally advanced colorectal cancer treated by preoperative chemoradiotherapy. World J Gastroenterol 2014;20:2023-2029

56. Maas M, Beets-Tan RG, Lambregts DM, Lammering G, Nelemans PJ, Engelen SM, et al. Wait-and-see policy for clinical complete responders after chemoradiation for rectal cancer. J Clin Oncol 2011;29:4633-4640

57. Maas M, Lambregts DM, Nelemans PJ, Heijnen LA, Martens $\mathrm{MH}$, Leijtens JW, et al. Assessment of clinical complete response after chemoradiation for rectal cancer with digital rectal examination, endoscopy, and MRI: selection for organsaving treatment. Ann Surg Oncol 2015;22:3873-3880

58. Barbaro B, Vitale R, Leccisotti L, Vecchio FM, Santoro L, Valentini V, et al. Restaging locally advanced rectal cancer with MR imaging after chemoradiation therapy. Radiographics 2010;30:699-716

59. van der Valk MJM, Hilling DE, Bastiaannet E, MeershoekKlein Kranenbarg E, Beets GL, Figueiredo NL, et al.; IWWD Consortium. Long-term outcomes of clinical complete responders after neoadjuvant treatment for rectal cancer in the International Watch \& Wait Database (IWWD): an international multicentre registry study. Lancet 2018;391:2537-2545

60. Park SH, Lim JS, Lee J, Kim HY, Koom WS, Hur H, et al. Rectal mucinous adenocarcinoma: MR imaging assessment of response to concurrent chemotherapy and radiation therapy-A hypothesis-generating study. Radiology 2017;285:124-133

61. Sclafani F, Brown G, Cunningham D, Wotherspoon A, Mendes LST, Balyasnikova S, et al. Comparison between MRI and pathology in the assessment of tumour regression grade in rectal cancer. Br J Cancer 2017;117:1478-1485

62. Kim S, Han K, Seo N, Kim HJ, Kim MJ, Koom WS, et al. T2weighted signal intensity-selected volumetry for prediction of pathological complete response after preoperative chemoradiotherapy in locally advanced rectal cancer. Eur Radiol 2018;28:5231-5240
63. Kluza E, Rozeboom ED, Maas M, Martens M, Lambregts DM, Slenter J, et al. T2 weighted signal intensity evolution may predict pathological complete response after treatment for rectal cancer. Eur Radiol 2013;23:253-261

64. Kim SH, Lee JM, Hong SH, Kim GH, Lee JY, Han JK, et al. Locally advanced rectal cancer: added value of diffusionweighted MR imaging in the evaluation of tumor response to neoadjuvant chemo- and radiation therapy. Radiology 2009;253:116-125

65. Lambregts DM, Vandecaveye V, Barbaro B, Bakers FC, Lambrecht M, Maas M, et al. Diffusion-weighted MRI for selection of complete responders after chemoradiation for locally advanced rectal cancer: a multicenter study. Ann Surg Oncol 2011;18:2224-2231

66. Curvo-Semedo L, Lambregts DM, Maas M, Thywissen T, Mehsen RT, Lammering $G$, et al. Rectal cancer: assessment of complete response to preoperative combined radiation therapy with chemotherapy--conventional MR volumetry versus diffusionweighted MR imaging. Radiology 2011;260:734-743

67. Gerard JP, Romestaing P, Chapet 0. Radiotherapy alone in the curative treatment of rectal carcinoma. Lancet Oncol 2003;4:158-166

68. Habr-Gama A, Perez R0, Proscurshim I, Campos FG, Nadalin W, Kiss $D$, et al. Patterns of failure and survival for nonoperative treatment of stage $\mathrm{c} 0$ distal rectal cancer following neoadjuvant chemoradiation therapy. J Gastrointest Surg 2006;10:1319-1328; discussion 1328-1329

69. Duldulao MP, Lee W, Streja L, Chu P, Li W, Chen Z, et al. Distribution of residual cancer cells in the bowel wall after neoadjuvant chemoradiation in patients with rectal cancer. Dis Colon Rectum 2013;56:142-149

70. Paty PB, Nash GM, Baron P, Zakowski M, Minsky BD, Blumberg $D$, et al. Long-term results of local excision for rectal cancer. Ann Surg 2002;236:522-529; discussion 529-530

71. Kim JG, Song KD, Kim SH, Kim HC, Huh JW. Diagnostic performance of MRI for prediction of candidates for local excision of rectal cancer (ypT0-1N0) after neoadjuvant chemoradiation therapy. J Magn Reson Imaging 2016;44:471477

72. Madbouly KM, Remzi FH, Erkek BA, Senagore AJ, Baeslach $C M$, Khandwala $F$, et al. Recurrence after transanal excision of T1 rectal cancer: should we be concerned? Dis Colon Rectum 2005;48:711-719; discussion 719-721

73. Kim NK. Anatomic basis of sharp pelvic dissection for curative resection of rectal cancer. Yonsei Med J 2005;46:737-749 